\title{
UPON ELEMENT-FREE GALERKIN METHOD AND ITS USING IN STATIC AND DYNAMIC STRUCTURE ANALYSIS
}

\author{
Vasile NĂSTĂSESCU*, Vlad-Andrei BÂRSAN** \\ *Military Technical Academy, Bucharest, Romania \\ **"Lucian Blaga" University Sibiu, Romania \\ nastasescuv@gmail.com
}

\begin{abstract}
This paper presents, in a synthetically way, the fundamentals of the element-free Galerkin (EFG) method - a meshfree method - under development but with many capabilities for solving complex problems in mechanical engineering, like impact problems etc. For interpolation, the EFG method uses moving least-squares (MLS) interpolants in curve and surface fitting. Unlike other interpolants, the MLS interpolants do not pass through the data because the Dirac function properties are not available. This aspect could be a disadvantage of the EFG method but next to it, there are many advantages. Upon these issues a discussion exists in this paper. Finally, some applications of the EFG method are presented referring to static and dynamic analysis of structures. The examples and conclusions can be useful for knowing and using of the EFG method.
\end{abstract}

Keywords: EFG, moving least-squares interpolants, meshfree, weight function

\section{Introduction}

Among numerical methods for structure analysis, relatively new methods appeared, it is about meshfree or meshless methods. A definition of an meshfree method (G.R. Liu, 2002) says that this establishes an algebraic equation system for the whole problem domain without using a predefined mesh for the domain discretization. This is something completely different from finite element method (FEM).

Ideally, a meshfree or meshless method should not ask for any mesh. From this point of view, smoothed particle hydrodynamics (SPH) method is a pure meshless or meshfree method.

The element-free Galerkin is a meshless or a meshfree method in the sense of the above general definition.

The meshfree methods use a set of nodes scattered within the problem domain. These nodes don't represent a discretization of the problem domain. Such nodes do not form a mesh and they are called field nodes.

Because the literature makes a difference between interpolation and approximation, it is necessary to notice that EFG method, unlike the FEM, uses the approximation. This means that by approximation procedure do not return nodal function values. By interpolation, the approximation procedure reproduces the exact values of the approximated function at the nodes.

The formulation of an equation set, by one or other numerical method can be made in a strong or weak form.

In a strong-form formulation, the approximate unknown function (e.g. u, v etc.) should have sufficient degree of consistency, so that it is differentiable up to the order of the partial differential equations.

In a weak-form formulation, the approximate unknown function (e.g. u, v 
etc.) has a weaker consistency, by introducing an integral operation to the system equations based on a mathematical or physical principle.

Obtaining the exact solution, by a strongform formulation of an equation system is ideal, but often very difficult.

As the weak-form formulation is based on global or local domain, we must distinguish between global or local weak-forms. From this point of view, the EFG method is a global weak-form method.

\section{Moving least-squares interpolant}

The Element-free Galerkin method uses the moving least-squares approximation of a function $u(x)$ representing a field variable. The approximated value of $u(x)$ will be denoted by $u^{h}(x)$ defined by expression:

$$
u^{h}(x)=\sum_{i=1}^{n} H_{i}(x) \cdot b_{i}(x)
$$

In a matrix form is written:

$$
u^{h}(x)=\mathrm{H}^{\mathrm{T}}(\mathrm{x}) \mathrm{b}(\mathrm{x})
$$

where $n$ is the order of the completeness in this approximation, the monomial $H_{i}(x)$ are basis functions and $b_{i}(x)$ are the coefficients of the approximation.

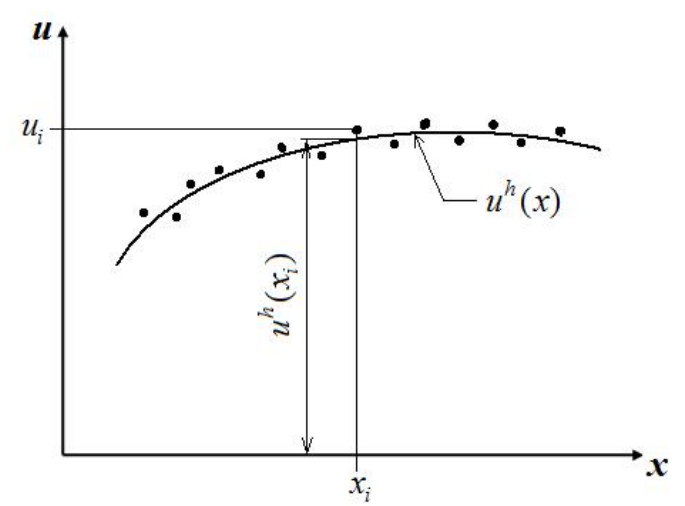

Fig. 1. Nodal parameters $u_{i}$ and approximate

$$
\text { function } u^{h}\left(x_{i}\right)
$$

As the Figure 1 shows, in the moving leastsquares approximation it is a difference between nodal parameter and its approximated value for a node $i$.

The coefficients $b_{i}(x)$ for a point $\mathbf{x}$ depend on the sampling points $\mathbf{x}_{\mathbf{I}}$ which are selected by a weighting function $w_{a}\left(\mathbf{x}-\mathbf{x}_{\mathbf{I}}\right)$.

A weighting function is defined on a compact support defined by a measure " $a$ " of a sub-domain. Each sub-domain $\Omega_{I}$ is associated with a node $I$. Often a such subdomain is a ball, like in the Figure 2.

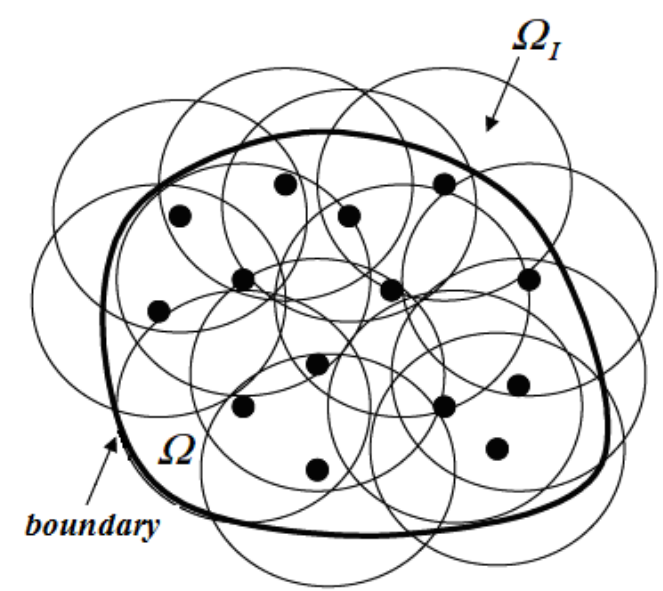

Fig. 2. A mesh-free discretization

The moving least-squares technique is based on minimizing the weighted $\mathrm{L}_{2}$-Norm $(J)$ defined by the relation (3) or (4); NP is the number of nodes (points) within the support domain where $w_{a}\left(\mathbf{x}-\mathbf{x}_{\mathbf{I}}\right) \neq 0$.

$$
\begin{aligned}
& J=\sum_{I=1}^{N P} W_{a}(x)\left(x-x_{I}\right)\left[u^{h}(x)-u_{i}\left(x_{I}\right)\right]^{2} \\
& J=(\mathbf{H b}-\mathbf{u})^{\mathrm{T}} \mathrm{W}_{\mathrm{a}}(\mathbf{x})(\mathbf{H} \mathbf{b}-\mathbf{u})
\end{aligned}
$$

In the relations (3) and (4) the following notations were used:

$$
\begin{aligned}
& \mathbf{u}^{\mathrm{T}}=\left(u_{1}, u_{2}, \ldots, u_{N P}\right) \\
& \mathbf{H}=\left[\begin{array}{l}
\left\{\mathrm{H}\left(\mathrm{x}_{1}\right)\right\}^{\mathrm{T}} \\
\ldots \\
\left\{\mathrm{H}\left(\mathrm{x}_{\mathrm{NP}}\right)\right\}^{\mathrm{T}}
\end{array}\right] \\
& \left\{\mathrm{H}\left(\mathrm{x}_{\mathrm{i}}\right)\right\}^{\mathrm{T}}=\left\{\mathrm{H}_{1}\left(\mathrm{x}_{\mathrm{i}}\right), \ldots, \mathrm{H}_{\mathrm{n}}\left(\mathrm{x}_{\mathrm{i}}\right)\right\} \\
& \mathrm{W}_{\mathrm{a}}=\operatorname{diag}\left[\mathrm{w}_{\mathrm{a}}\left(\mathrm{x}-\mathrm{x}_{1}\right), \ldots, \mathrm{w}_{\mathrm{a}}\left(\mathrm{x}-\mathrm{x}_{\mathrm{NP}}\right)\right]
\end{aligned}
$$

The coefficients $\mathbf{b}$ result from equation: 


$$
\frac{\partial \mathrm{J}}{\partial \mathrm{b}}=\mathrm{M}^{[\mathrm{n}]}(\mathrm{x}) \mathrm{b}(\mathrm{x})-\mathrm{B}(\mathrm{x}) \mathrm{u}=0
$$

where,

$$
\begin{aligned}
& M^{[n]}(x)=H^{T} W_{a}(x) H \\
& B(x)=H^{T} W_{a}(x)
\end{aligned}
$$

resulting:

$$
\mathrm{b}(\mathrm{x})=\mathrm{M}^{[\mathrm{n}]^{-1}}(\mathrm{x}) \mathrm{B}(\mathrm{x}) \mathrm{u}
$$

Using the solution of the equations (1), (10), (11) and (12) the EFG approximation is obtained:

$$
u^{h}(x)=\sum_{I=1}^{N P} \Psi_{I}(x) u_{I}
$$

$\Psi_{I}(x)$ are shape functions having the expressions:

$$
\Psi_{\mathrm{I}}(\mathrm{x})=\mathrm{H}^{\mathrm{T}}(\mathrm{x}) \mathrm{M}^{[\mathrm{n}]^{-1}}(\mathrm{x}) \mathrm{B}(\mathrm{x})
$$

\section{Choice of weight function}

The choice of the weight function can be theoretically arbitrary as long as these meet some conditions. Synthetically, the most important conditions are:

- to be greater zero within the support domain;

- to be zero outside the support domain;

- to be monotonically decreasing from the point of interest;

- to be sufficient smooth, especially on the boundary.

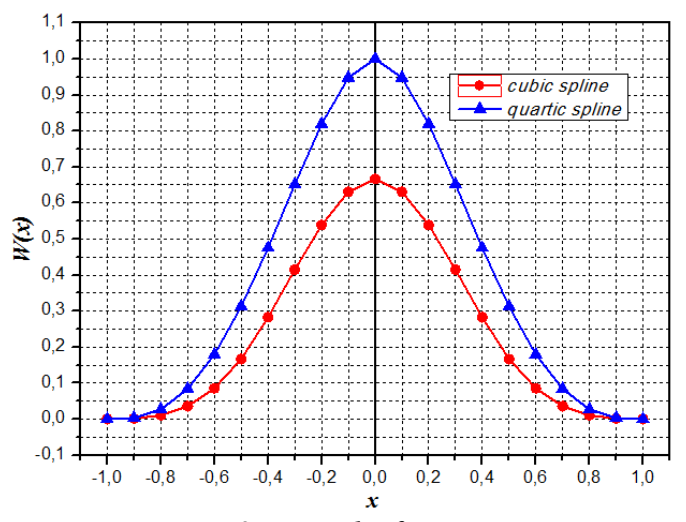

Fig. 3. Weight functions

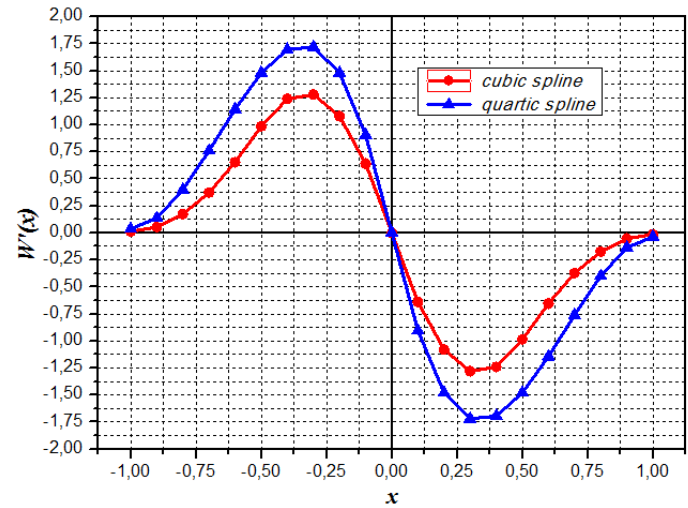

Fig. 4. First derivatives of weight functions

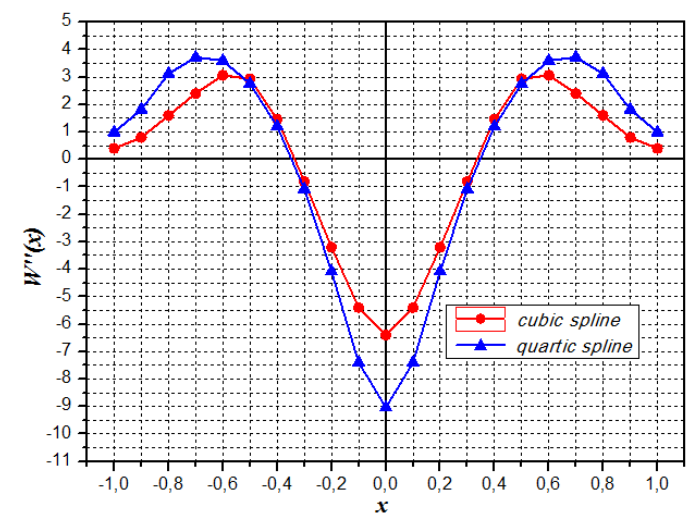

Fig. 5. The second derivatives of weight functions

The most used weight functions are: the cubic and the quartic spline functions.

In the Figure 3 a graphical representation of these weight functions is presented.

The Figures 4 and 5 show the first and respectively the second derivatives of the same weight functions presented in the Figure 3.

\section{The matrix equation system}

The moving least-squares approximation lacks the Kronecker delta function property. A weak-form formulation, including all the loads (on domain and on boundaries) is:

$$
\begin{aligned}
& \int_{\Omega} \delta(\mathrm{Lu})^{\mathrm{T}} \mathrm{D}(\mathrm{Lu}) \mathrm{d} \Omega=\int_{\Omega} \delta \mathrm{u}^{\mathrm{T}} \mathrm{bd} \Omega+\int_{\Gamma_{\mathrm{t}}} \delta \mathrm{u}^{\mathrm{T}} \overline{\mathrm{t}} \mathrm{d} \Gamma+ \\
& +\delta \int_{\Gamma_{\mathrm{u}}} \frac{1}{2}(\mathrm{u}-\overline{\mathrm{u}})^{\mathrm{T}} \alpha(\mathrm{u}-\overline{\mathrm{u}}) \mathrm{d} \Gamma
\end{aligned}
$$

In the relation (15), the used notations, for a 2D problem, have the following forms and meaning: 
$\mathbf{L}=\left[\begin{array}{cc}\frac{\partial}{\partial x} & 0 \\ 0 & \frac{\partial}{\partial y} \\ \frac{\partial}{\partial y} & \frac{\partial}{\partial x}\end{array}\right]:$ differential operator;

$\mathrm{u}^{\mathrm{T}}=\left\{\begin{array}{ll}\mathrm{u} & \mathrm{v}\end{array}\right\}$ : displacement vector;

$\mathrm{D}=\frac{E}{1-v^{2}}\left[\begin{array}{ccc}1 & v & 0 \\ v & 1 & 0 \\ 0 & 0 & \frac{1-v}{2}\end{array}\right]:$ matrix of material constants, for a plane stress state;

$b^{T}=\left\{\begin{array}{ll}b_{x} & b_{y}\end{array}\right\}$ : the body force vector;

$\overline{\mathrm{t}}=\sigma \cdot \mathrm{n}$ : the prescribed traction on the boundary $\left(\Gamma_{t}\right)$;

$\mathrm{n}$ : the vector of unit outward normal at a point on boundary;

$\overline{\mathrm{u}}=\mathrm{u}$ : the prescribed displacement on the boundary $\left(\Gamma_{u}\right)$;

$\alpha=\left[\begin{array}{llll}\alpha_{1} & \alpha_{2} & \ldots & \alpha_{k}\end{array}\right]$ : is a diagonal matrix of penalty factors, where $k=2$ for $2 \mathrm{D}$ and $k=3$ for $3 \mathrm{D}$; the penalty factors $\alpha_{i}$ can be function of coordinates (different from each other), but they have to be given. Practically, a constant large positive number is used.

Taking into account the fundamentals of the EFG method presented above, by their introducing in the relation (15), the following matrix equation is obtained:

$\left\lfloor K+K^{\alpha}\right\rfloor \cdot U=F+F^{\alpha}$

$K^{\alpha}$ is the global penalty stiffness matrix and $F^{\alpha}$ is an additional force vector.

In the case of using of the Lagrange multiplier method for essential boundary conditions, the relation (15) will be rewritten and penalty factors $a_{i}$ will be changed with Lagrange multiplayers $\lambda$, as it is shown in the relation (17).

$$
\begin{aligned}
& \int_{\Omega}(L \delta u)^{T} \cdot(D L u) \cdot d \Omega=\int_{\Omega} \delta u^{T} \cdot b \cdot d \Omega+ \\
& +\int_{\Gamma_{t}} \delta u^{T} \cdot \bar{t} \cdot d \Gamma+\int_{\Gamma_{u}} \delta \lambda^{T} \cdot(u-\bar{u}) \cdot d \Gamma- \\
& -\int_{\Gamma_{u}} \delta u^{T} \cdot \lambda \cdot d \Gamma
\end{aligned}
$$

By a lot of mathematical transformation, by taking into account the relations of the EFG fundamentals, finally the following matrix equation is obtained,

$\left[\begin{array}{cc}K & G \\ G^{T} & 0\end{array}\right] \cdot\left[\begin{array}{c}U \\ \Lambda\end{array}\right]=\left[\begin{array}{l}F \\ Q\end{array}\right]$

where $K$ is the global stiffness matrix, $G$ is the global stiffness matrix resulting from boundary conditions, $U$ is the vector of the nodal parameters of the displacements, $\Lambda$ is a vector collecting the nodal Lagrange multipliers for all field nodes on the boundaries, $F$ is the global force vector and $Q$ is the global vector of the forces resulting from the prescribed displacement on the boundary.

The equations (16) and (18) represent the final discretized system equations for the EFG method, using penalty method and Lagrange multiplier method, respectively.

Solving of the equation (16) or (18) nodal parameters of the displacements are obtained; then, the nodal displacements $u^{h}$ are obtained.

\section{Numerical results}

Some numerical results will be presented and analysed for two comparative examples of a simple structure under static and dynamic loads.

\subsection{Cantilever beam under static load}

This structure is presented in the Figure 6. Firstly, an analytical solution is presented. The maximum stress is:

$$
\sigma_{x \max }=\frac{M_{\text {max }}}{W_{z}}=250 \mathrm{MPa}
$$

The used material has Young modulus $E=2 \cdot 10^{5} \mathrm{MPa}$ and Poisson ratio zero. 


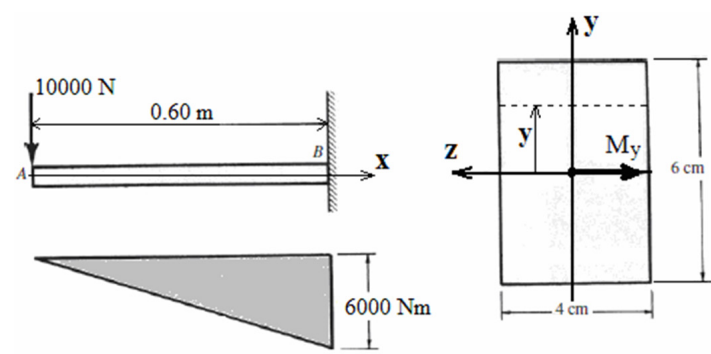

Fig. 6. Problem formulation

The maximum deflection is:

$f_{\text {max }}=\frac{F l^{3}}{3 E I_{z}}=-0.005 \mathrm{~m}$

Numerical analysis by FEM and EFG was performed using the model presented in the Figure 7, consisting of 9331 nodes and 9000 elements with four nodes/element.

Fig. 7. The model for numerical analysis
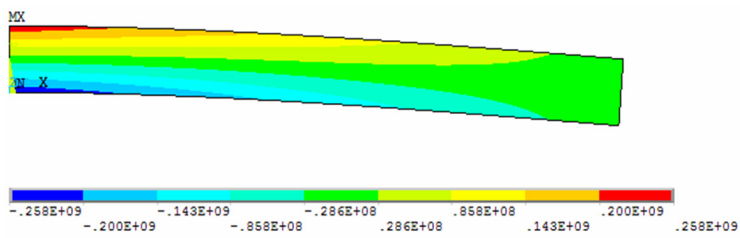

Fig. 8. The $\sigma_{x}$ stress field on the deformed state of the beam, by FEM

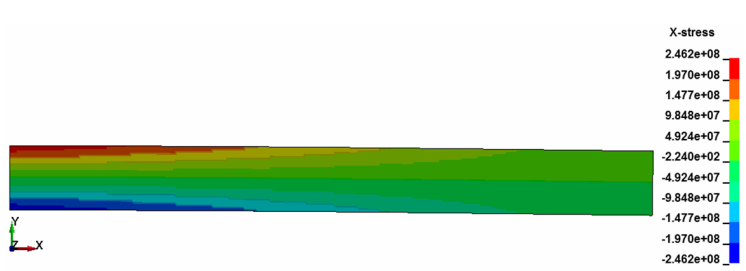

Fig. 9 The $\sigma_{x}$ stress field of the beam, by EFG
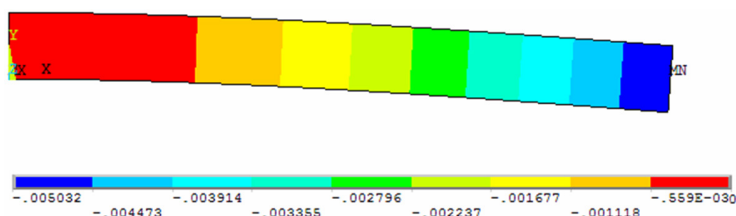

Fig. 10. The deformed state of beam by FEM

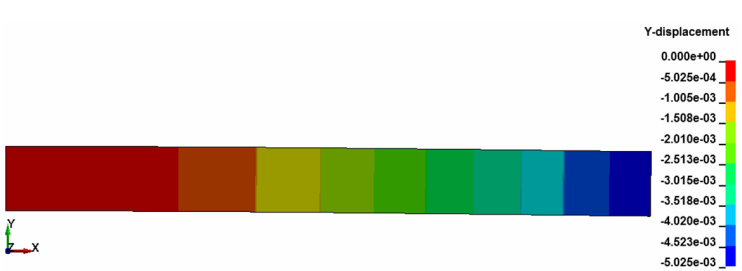

Fig. 11. Deflection of the beam by EFG
Table 1. Results and errors

\begin{tabular}{|c|c|c|c|}
\hline \multirow[b]{2}{*}{$\begin{array}{l}\text { Calculated } \\
\text { parameters }\end{array}$} & \multirow{2}{*}{$\begin{array}{l}\text { Analytical } \\
\text { result }\end{array}$} & \multicolumn{2}{|c|}{ 2D Model } \\
\hline & & FEM & $\begin{array}{c}\text { ELG } \\
\text { method }\end{array}$ \\
\hline \multirow{3}{*}{$\begin{array}{c}\left(\sigma_{x}\right)_{\max } \\
{[\mathrm{MPa}]}\end{array}$} & \multirow{3}{*}{250,00} & 258 & 246,2 \\
\hline & & \multirow{2}{*}{$\begin{array}{c}E r .: \\
3,20 \%\end{array}$} & $\begin{array}{c}E r .: \\
-1,52 \% \\
\end{array}$ \\
\hline & & & $\begin{array}{l}E r_{F E M}: \\
-4.57 \%\end{array}$ \\
\hline \multirow{3}{*}{$\begin{array}{c}f_{\max } \\
{[\mathrm{m}]}\end{array}$} & \multirow{3}{*}{0,0050} & 0,005032 & 0,005025 \\
\hline & & \multirow{2}{*}{$\begin{array}{c}E r .: \\
0,64 \%\end{array}$} & $\begin{array}{c}E r .: \\
0,50 \%\end{array}$ \\
\hline & & & $\begin{array}{l}E r_{F E M}: \\
-0,14 \%\end{array}$ \\
\hline
\end{tabular}

The results regarding maximum $\sigma_{x}$-stress and maximum deflection of the beam are comparatively presented in the above table.

\subsection{The impact of the ball with a plate}

The impact ball-plate is numerically simulated by FEM and by EFG method. The numerical model, used for both method is presented in the Figure 12.

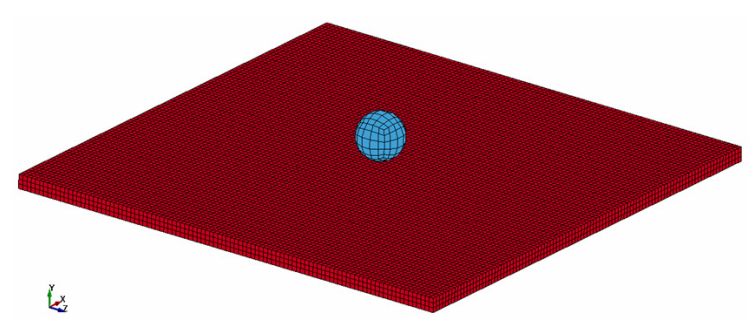

Fig. 12. The model for FEM and EFG method

This uses 3D SOLID finite elements with 8 nodes. The plate is modelled by 30000 finite elements and 40804 nodes (FE size is $1 \mathrm{~mm})$. The ball consists in 875 finite elements and 976 nodes. Constraints were applied only for the ball, consisting in the blocking of all displacement, excepting the Y-displacement (impact direction). The impact velocity was $420 \mathrm{~m} / \mathrm{s}$. For the plate the plastic-kinematic material model was used and for the ball the rigid material model was used. The characteristics of the materials (aluminum for plate and steel for ball) are presented in the Table 2. Diameter of the ball is $0.01 \mathrm{~m}$; the plate dimensions are $100 \times 100 \times 3 \mathrm{~mm}$. The time analysis was of $45 \cdot 10^{-6}[\mathrm{~s}]$, adopted for a complete perforation of the plate. 
Table 2. Material characteristics

\begin{tabular}{|l||l|c|}
\hline \multicolumn{1}{|c||}{ Material characteristics } & \multicolumn{1}{c|}{ Plate } & \multicolumn{1}{c|}{ Ball } \\
\hline \hline Density $\left[\mathrm{kg} / \mathrm{m}^{3}\right]$ & 2700 & 7850 \\
\hline Young modulus $[\mathrm{MPa}]$ & $0.7 * 10^{5}$ & $2.1 * 10^{5}$ \\
\hline Poisson ratio & 0.33 & 0.29 \\
\hline Yielding stress $[\mathrm{MPa}]$ & 200 & - \\
\hline Failure strain & 0.75 & - \\
\hline
\end{tabular}
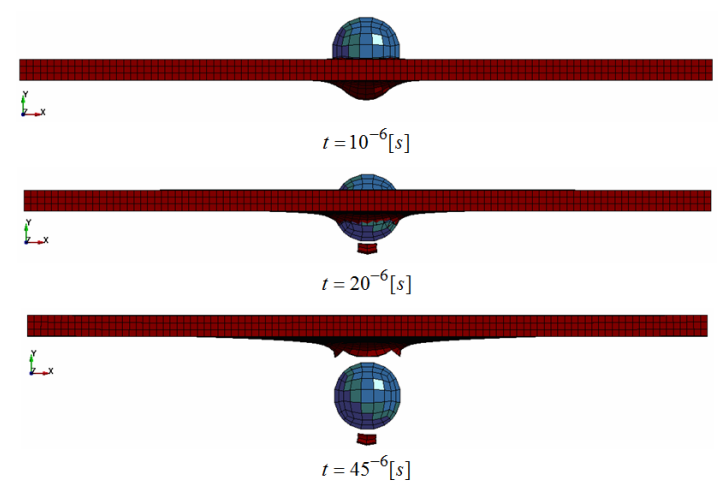

Fig. 13 The penetration evolution by FEM

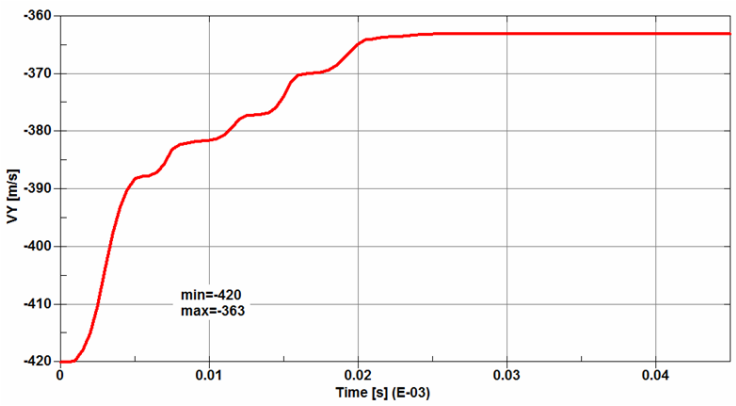

Fig. 15 Time evolution of ball velocity by FEM

Table 3. Comparative results

\begin{tabular}{|l||c|c|}
\hline \multicolumn{1}{|c||}{ Results } & FEM & $\begin{array}{c}\text { EFG } \\
\text { Method }\end{array}$ \\
\hline \hline $\begin{array}{l}\text { Maximum ball } \\
\text { displacement [m] }\end{array}$ & \multirow{2}{*}{0.016776} & 0.017045 \\
\cline { 3 - 3 } $\begin{array}{l}\text { Residual ball } \\
\text { velocity [m/s] }\end{array}$ & \multirow{E}{*}{363.00} & $\mathbf{3 7 6 0 \%}$ \\
\hline $\begin{array}{l}\text { Maximum von } \\
\text { Mises stress [Pa] }\end{array}$ & $2.00 * 10^{8}$ & $\mathbf{E r . : 2 . 3 4 \%}$ \\
\cline { 3 - 3 } & & $\mathbf{E r . : - 2 , 3 5 \%} 10^{8}$ \\
\hline
\end{tabular}

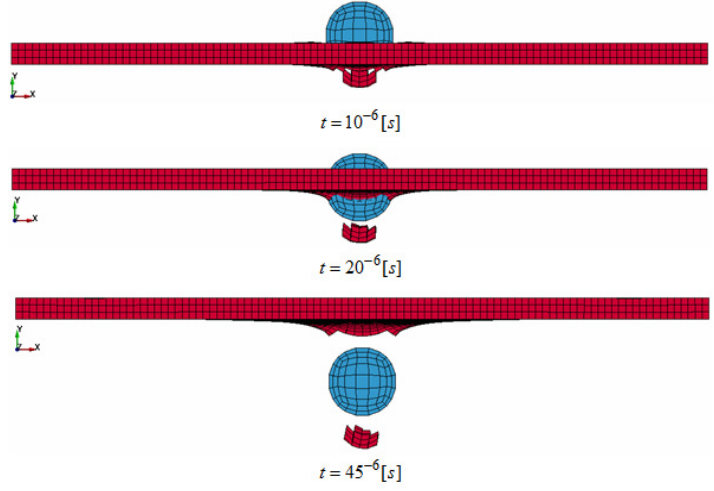

Fig. 14 The penetration evolution by EFG

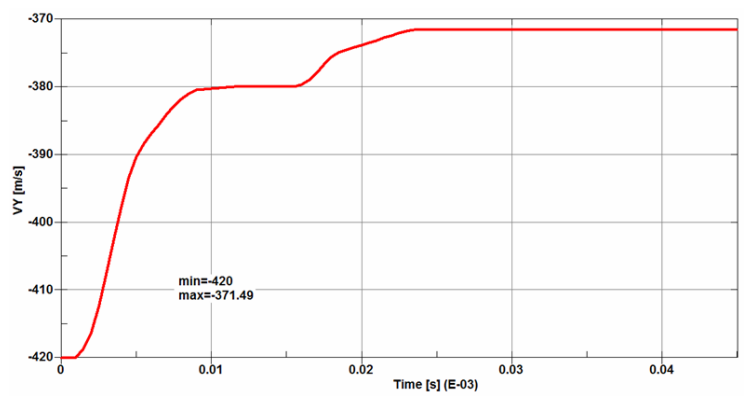

Fig. 16 Time evolution of ball velocity by EFG

\section{Conclusions}

The EFG method is an available tool for static and dynamic structure analysis. This relativelly new method has all those advantages of mesh-free methods. Among these, the most important advantage is a better accuracy in stress calculus (Table 1). The results obtained by EFG method are very closed with the FEM results (Table 3). The main shortcomings of EFG can be a computation speed (slower than FEM) and its development stage (beginning).

The EFG method is implemented in many power and professional programs, but commercial dedicated software packages only a few exist.

\section{References}

[1] Hallquist, O. John, LS-DYNA Theory Manual, Livermore, California 94551, 2006,

[2] Liu, L., Chua, L.P., Ghista, D.N., Element-free Galerkin Method for Static and Dynamic Analysis of Spatial Shell Structures, Journal of Sound and Vibration 295 (2006) 388-406, www.elsevier.com/locate/jsvi

[3] Liu, G.R., Gu, Y.T., An Introduction to Meshfree Methods and Their Programming, Published by Springer, P.O. Box 17, 3300 AA Dordrecht, The Netherlands, 2005

[4] Năstăsescu, V., Ştefan, A., Lupoiu, C., Analiza neliniară a structurilor mecanice, Editura Academiei Tehnice militare, Bucureşti, 2002 